\title{
Reasoning about actions with imprecise and incomplete state descriptions
}

\author{
Célia da Costa Pereira, Andrea G.B. Tettamanzi* \\ Dipartimento di Tecnologie dell'Informazione, Università degli Studi di Milano, Via Bramante 65, I-26013 Crema (CR), Italy
}

Available online 30 November 2008

\begin{abstract}
This article is a first step in the direction of extending possibilistic planning to account for incomplete and imprecise knowledge of the world state. Fundamental definitions are given and the possibilistic planning problem is recast in this new setting. Finally, it is shown that, under certain conditions, possibilistic planning with imprecise and incomplete state descriptions is no harder than possibilistic planning with crisp and complete information.
\end{abstract}

(C) 2008 Elsevier B.V. All rights reserved.

PACS: 07.05.M

Keywords: Planning; Possibility theory; Neutrosophy

\section{Introduction}

Planning is a branch of artificial intelligence which studies how to find the most suitable sequence of actions to take a system from a given initial state into a desired state, called goal.

In a classical planning problem, it is assumed that actions are deterministic, the initial state is known, and the goal is defined by a set of final states; a solution plan is then an unconditional sequence of actions that leads from the initial state to a goal state. However, most practical problems do not satisfy these assumptions of complete and deterministic information.

Classical planners, like NONLIN [26], TWEAK [5], or UCPOP [18], assume correct and complete information about the world, i.e., that part of reality relevant to the planning problem that is to be solved. Having complete and correct information makes planning more affordable, since the planning agent need not obtain information from the external world - all relevant information is present in the agent's world model (closed world assumption). However, in many realistic cases, an agent may not have a perfect description of the world. Three sources of "imperfection" exist:

(1) incompleteness - information about the world state does not cover all relevant details: in a propositional settings, this amounts to saying that, for a given atomic proposition $p$, neither $p$ nor $\neg p$ is asserted in the planner's knowledge base;

(2) uncertainty - the outcome of a given action, executed in a given world state, cannot be predicted with certainty; instead, a number of distinct outcomes, or effects, are possible: for instance, the action "open the door" may result

\footnotetext{
* Corresponding author.

E-mail addresses: celia.pereira@unimi.it (C. da Costa Pereira), andrea.tettamanzi@unimi.it (A.G.B. Tettamanzi).
} 
in the door being open, if the door is unlocked, but may also result in the door being still closed, if the door is locked, which the planner does not know in advance.

(3) imprecision-some relevant details of a world state are described in imprecise, or vague, terms: e.g., "the door is near", or "the battery is low";

Planning under incompleteness and/or uncertainty has been widely investigated in the AI literature. Most of these approaches, like Buridan [16], POSPLAN [7], Conformant Graphplan [22], CNLP [19], and Cassandra [21], extend algorithms/systems devised for classical planning. The latter two planners deal with conditional planning in which the sequence of actions to be executed depends on dynamic conditions, while the former four planners can solve secure planning. On the other hand, in [11], a new language has been proposed for dealing with incomplete information. In [2], the authors provide a model to capture the evolution of the agent's knowledge as it engages in the activities of planning and execution. In [28], Graphplan [4] has been extended to handling uncertainty and sensing actions. Similarly, in [20], a new approach has been proposed to planning with incomplete information and sensing, based on a higher-level, "knowledge-based", representation of the planner's knowledge and of the domain actions. In particular, the authors use a set of formulas from a first-order modal logic of knowledge to represent the planner's incomplete knowledge state. A similar approach has been proposed in [15], which provides a new technique for generating cyclic plans in a KL-based framework.

Other approaches, like [17,13,22], propose the use of automated-reasoning techniques for planning under incomplete knowledge. The works proposed by Eiter et al. [11] and Guinchiglia [14] differ considerably from previous approaches, in the sense that they use deduction techniques for solving planning tasks under incompletness instead of extending classical algorithms/systems.

Imprecision of the information about the world state is little considered in the literature, as most works assume the planning agent disposes of correct information. Imprecise knowledge is essentially dealt within the domain of fuzzy control [24].

In this article, we propose an original approach which is a first step toward further extending a possibilistic approach [7] to obtain a general formalism for handling incompleteness, uncertainty, and imprecision in a uniform way. The aim of the formalism is to make it possible to represent dynamical aspects of the world state description in the presence of incompletness, imprecision, and uncertainty, and to make it possible to deal with planning problems in a sensible way while only taking into account available knowledge.

The work presented in this paper is an extension of the possibilistic approach to planning proposed in [7] which, in turn, is an extension of the well-known STRIPS [12] formalism to allow the representation of possibilistic uncertainty.

The article is organized as follows: possibilistic planning is introduced in Section 2; Section 3 discusses the use of fuzzy state descriptions to represent imprecise and incomplete knowledge about the world state and provides a model-theoretic semantics and useful properties for such representation; Section 4 defines the syntax and semantics of possibilistic actions in this framework; the notion of plan is recast in the fuzzy possibilistic setting in Section 5, and a definition and characterization of fuzzy possibilistic planning problem is provided in Section 6. Section 7 concludes.

\section{Possibilistic planning}

Let us first of all focus on the benefits of using possibility theory in planning, especially in comparison with probabilistic planning [7]:

- Possibility theory is an ordinal model: only the order induced by possibility distributions is important, not the precise values of the degrees. This ordinal aspect of a possibilistic representation (contrarily to probabilistic representations which are intrinsically quantitative) gives the model more robustness to imprecision on the degrees and is thus particularly suited to cases where there is a lack of statistical data.

- Actions with possibilistic transition functions $\pi[. \mid s, a]$ are a graded generalization of nondeterministic actions, so that possibilistic planning encompasses nondeterministic planning as a particular case (obtained by allowing only 0 and 1 as possibility degrees). This is not the case with probability theory, at least if we work with a single probability distribution as in Bayesian approaches: there is no mean of encoding graded nondeterminism by a probability distribution. 
The possibilistic approach to planning proposed in [7], which is the starting point of this work, considers the class of planning problems in which

(1) the environment is static, which means all changes that take place in the world result from actions specified in the plan given by the agent;

(2) information about the world state is precise and complete; and

(3) the environment is assumed to be unobservable during plan execution, thus requiring the search for nonconditional plans that must be robust to uncertainty.

The approach can be regarded as a possibilistic counterpart of the probabilistic approach by Kushmerick et al. [16], in which possibility distributions are used to represent the uncertainty both on the initial and subsequent states and on the outcomes of the execution of an action in a particular context. Two notions of solution plans for such a possibilistic planning problem are introduced:

- $\gamma$-acceptable plans, that lead to a goal state with a certainty greater than a given threshold $\gamma$ and

- optimally safe plans, that lead to a goal state with maximal certainty.

The main benefit expected from the adoption of a framework based on possibility theory [9] is the ability to represent qualitatively, and thus more faithfully, what is known about the initial state and the possible effects of actions; the possibilistic approach is likely to be less sensitive to a lack of precision in the assessment of uncertainty. Using a model in which actions have possibilistic effects is particularly well suited for cases in which the probabilities of the resulting effects of actions are not available, not very reliable, or hard to obtain, that is, in situations of partial or total ignorance about the immediate consequences of applying an action. Moreover, the notion of action with possibilistic effects properly generalizes the notion of nondeterministic actions by enabling the representation of ordinal grading in the uncertainty that characterize the uncontrollable choice process through which the real effect of an action will be determined. What is represented is simply that one or several effects are normal in essence (nothing prevents them from occurring) and that some are more normal (less exceptional) than others, that is, some may be considered more plausible than others in the absence of further information.

One of the most important results of the above approach is that the search of a $\gamma$-acceptable plan amounts to solving a planning problem straightforwardly derived from the original possibilistic problem and consisting only of pure (nongraded) nondeterministic actions.

With respect to the above starting point [7]:

- we keep the assumption that the environment is static;

- we keep the assumption that the environment is unobservable during plan execution;

- we drop the closed world assumption; we adopt instead the open world assumption, stating that lack of knowledge about a proposition does not necessarily imply its falsity; therefore, the representation of world states is incomplete;

- we also drop the assumption that information about the world state is always precise: states are thus described by fuzzy literals. Therefore, a world state is a fuzzy interpretation of the propositions relevant to the planning problem.

We will employ, throughout the rest of the article, an example consisting of a simple planning problem in the domain of agronomy. A crop has to be grown in a field with the goal of obtaining a desired yield; depending on the conditions of the soil and of the weather, a farmer may apply several actions like sowing a standard or resistant strand of seeds, treating the field with pesticides, or harvesting.

\section{World state representation}

The first step in laying out a general formalism to handle incompleteness, uncertainty, and imprecision for reasoning about actions consists of deciding how to represent the world and its states.

We adopt an objectivist ontology whereby a real world is assumed to exist independently of the perceptions of a planning agent. At each point in time, the world state is completely, certainly, and precisely defined. However, in the spirit of abstracting away from irrelevant details, we do allow the world state to be identified imprecisely, in the technical sense stated in the Introduction, i.e., by means of propositions whose truth may be graded. Accordingly, a natural choice is to represent an objective world state as a complete assignment of truth degrees to all propositions defined for the problem at hand, i.e., a fuzzy interpretation. 
The above considerations apply to the representation of objective states of the world. A completely different story is the representation of a planning agent's knowledge about the world state. One might call this a subjective world state, but we prefer to call it a world state description. Based on the assumptions stated in Section 2, such a description may be incomplete, imprecise, and even uncertain, due to the unobservability assumption. Furthermore, the possibility of contradictory information, arising from conflicting sources (i.e., sensors) for the initial state, or from the nondeterminism of actions for intermediate and final states, cannot be ruled out.

\subsection{Fuzzy interpretations}

To begin with, we define the notion of fuzzy interpretation over the set of atomic propositions as a means for representing objective world states, and we extend it to the set of propositional formulas.

Definition 1. A fuzzy interpretation is an assignment of truth degrees in $[0,1]$ to all atomic propositions (or atoms, for short) defined in the problem domain. Let $\mathcal{A}$ be a set of atoms; a fuzzy interpretation $\mathcal{I}$ is a function

$$
\mathcal{I}: \mathcal{A} \rightarrow[0,1],
$$

which assigns a truth degree $p^{\mathcal{I}} \in[0,1]$ to all atoms $p \in \mathcal{A}$.

We will use a convenient notational convention, especially for illustrating the examples, for the extensional representation of a fuzzy set $S$ over the universe of discourse $\mathcal{A}$ as

$$
S=\sum_{p \in \mathcal{A}} \frac{S(x)}{x}=\frac{S\left(p_{1}\right)}{p_{1}}+\frac{S\left(p_{2}\right)}{p_{2}}+\cdots,
$$

where $p_{i} \in \mathcal{A}$ for all $i$ and $S(p)$ is the membership degree of $p$ in $S$. This notation is nothing more than a formal device and the fractions do not have to be interpreted as divisions but just as ordered pairs, while the + does not stand for algebraic sum but rather for a function-theoretic union.

In our example, $\mathcal{A}$ might consist of the following atomic propositions:

$c$ the desired crop has been sown;

$f$ weather conditions are favorable;

$g$ the crop is growing as desired;

$p$ the crop is affected by pest;

$y$ the desired yield has been reached.

A fuzzy interpretation represents an actual state of the world. Given $\mathcal{A}=\{c, f, g, p, y\}$, an example of fuzzy interpretation might be $\mathcal{I}$ such that

$$
c^{\mathcal{I}}=1, \quad f^{\mathcal{I}}=0.8, \quad g^{\mathcal{I}}=0, \quad p^{\mathcal{I}}=0.2, \quad y^{\mathcal{I}}=0,
$$

which can be written, more compactly, as $\mathcal{I}=1 / c+0.8 / f+0.2 / p$.

A fuzzy interpretation $\mathcal{I}$ can naturally be extended to all propositions (i.e., well-formed formulas in propositional logic) as follows, for all propositions $p$ and $q$ :

$$
\begin{aligned}
\top^{\mathcal{I}} & =1, \\
\perp^{\mathcal{I}} & =0, \\
(\neg p)^{\mathcal{I}} & =1-p^{\mathcal{I}}, \\
(p \wedge q)^{\mathcal{I}} & =\min \left\{p^{\mathcal{I}}, q^{\mathcal{I}}\right\}, \\
(p \vee q)^{\mathcal{I}} & =\max \left\{p^{\mathcal{I}}, q^{\mathcal{I}}\right\}, \\
(p \supset q)^{\mathcal{I}} & =\min \left\{1,1-p^{\mathcal{I}}+q^{\mathcal{I}}\right\}, \\
(p \equiv q)^{\mathcal{I}} & =\min \left\{(p \supset q)^{\mathcal{I}},(q \supset p)^{\mathcal{I}}\right\} .
\end{aligned}
$$

Note that for the implication we decided to use Łukasiewicz's fuzzy implication operator. This leads to a definition of fuzzy entailment (see Definition 4) that is a sound generalization of classical entailment, thanks to the 
fact that it satisfies the Sinha-Dougherty axioms for fuzzy set inclusion [6]. However, this is not the only possible choice: other forms of implication could be used, but some of the results could be affected. Exploring the impact of various choices of implications is an interesting research issue which, however, goes beyond the scope of this work.

In the example, $\mathcal{I}$ completely represents a state of the world where the crop has been sown, the weather conditions are quite favorable but the crop is not growing at all and, in addition, it is slightly affected by pest; the desired yield has not been reached at all. While involving vague (i.e., linguistic) information, such representation of the state of the world is by no means uncertain or incomplete. According to $\mathcal{I}$, the truth of formula $(c \wedge \neg f) \supset g$ ("if the crop has been sown but weather conditions are unfavorable, the crop is growing as desired") would be given by

$$
\begin{aligned}
((c \wedge \neg f) \supset g)^{\mathcal{I}} & =\min \left\{1,1-(c \wedge \neg f)^{\mathcal{I}}+g^{\mathcal{I}}\right\} \\
& =\min \left\{1,1-\min \left\{c^{\mathcal{I}}, 1-f^{\mathcal{I}}\right\}+g^{\mathcal{I}}\right\} \\
& =\min \{1,1-\min \{1,1-0.8\}+0\} \\
& =\min \{1,1-0.2+0\}=0.8 .
\end{aligned}
$$

\subsection{Fuzzy state descriptions}

Assuming complete knowledge of the world is irrealistic for most real-world planning problems. Instead, we want to deal with partial, imprecise representations of world states.

Fuzzy set theory and fuzzy logic provide a mathematical framework for dealing with incomplete and imprecise information. Atanassov introduced what he calls "intuitionistic fuzzy sets" [1], a generalization of fuzzy sets to deal with incomplete information by means of a bipolar representation. A set of this type is defined by a pair of membership and nonmembership functions, respectively, $T$ and $F$, with

$$
T(x)+F(x) \leqslant 1
$$

for all element $x$. This constraint bars Atanassov's membership/nonmembership pairs from handling inconsistent knowledge.

An interesting proposal to handle inconsistency in fuzzy logic is due to Smarandache [25], under the name neutrosophy [23]. The issue of representing incomplete, imprecise, and possibly inconsistent information is particularly felt in fuzzy databases. It is indeed within that area of research that the idea of using neutrosophic fuzzy sets to represent inconsistent (in addition to incomplete and imprecise) knowledge has been proposed [27]. Neutrosophic fuzzy sets are obtained by relaxing the constraint in Eq. (10).

Inspired by that proposal, we choose to represent the knowledge that a planning agent has about the actual world state as a fuzzy set of literals (i.e., atoms or negated atoms). The membership of positive and negated atoms can be regarded as deriving from membership/nonmembership pairs in the sense of Atanassov. We will call $\mathcal{S}$ the set of all such fuzzy state descriptions.

Let $\mathcal{L}=\mathcal{A} \cup \mathcal{A}\urcorner$ be the set of literals constructed on $\mathcal{A}$. For all $s \in \mathcal{S}$ and literal $\ell \in \mathcal{L}, s(\ell)$ is the membership degree of $\ell$ in $s$. We will say a state description $s$ is consistent if and only if, for all $p \in \mathcal{A}, s(p)+s(\neg p) \leqslant 1$.

Intuitively, $s(\ell)$ is the degree to which the planning agent knows literal $\ell$ is (or will be) true in state $s$. Alternatively, one may regard a fuzzy state description $s$ as a fuzzy set of objective world states. The degree to which an actual, objective world state matches description $s$ is the degree to which that world state belongs in $s$.

The adoption of neutrosophic fuzzy sets for representing state descriptions stems from the basic idea that an agent may have distinct estimates of the truth of related literals, like $p$ and $\neg p$ which ultimately go back to the same atomic proposition. When such estimates are contradictory or in partial disagreement, the agent might try to resolve that disagreement, but that would mean to lose some information. To avoid information loss, we tolerate some inconsistency. To be sure, other approaches exist to obtain the same goal, notably bipolar possibilistic logic [3]. The adoption of this framework would lead to alternative approaches, whose study will make the object of further research. In fact, the bipolar representation we are using is but one particular type among a variety of alternatives (see Dubois and Prade's recent discussion [10] of the salient features of the main three types), namely the type Dubois and Prade call "dual bivariate". 
In order to evaluate the degree to which a fuzzy state description $s$ is satisfied by a given world state $\mathcal{I}$, which is a fuzzy interpretation, we must extend fuzzy interpretations to fuzzy state descriptions as well.

Definition 2. Let $\mathcal{I}$ be a fuzzy interpretation; for all $s \in \mathcal{S}$,

$$
s^{\mathcal{I}}=\min _{\ell \in \mathcal{L}} \max \left\{1-s(\ell), \min \left\{s(\ell), \ell^{\mathcal{I}}\right\}\right\}
$$

The rationale for this definition is a fuzzification of the reckoning that, under the open-world assumption, if $s$ does not contain a literal, any truth assignment made by $\mathcal{I}$ to that literal satisfies $s$; however, if $s$ contains a literal, only those interpretations for which that literal is true satisfy $s$.

Fuzzy state descriptions represent what an agent knows or believes about a world state. Knowledge about a state may be partial (the agent may have no clues as to the actual truth value of some propositions). Uncertainty about a world state may arise as a consequence of the application of indeterministic possibilistic actions.

In our example, a possible fuzzy state description might be

$$
s=\frac{1}{c}+\frac{0.5}{p}+\frac{1}{\neg p},
$$

which describes states of the world where the crop has definitely been sown and, as far as the planning agent knows, there is a fair possibility that the crop is affected by pest, while it may very well be that the crop is not affected by pest. The truth degrees of all the remaining propositions are not specified, i.e., they may take up whatever value.

The degree to which $s$ would describe (i.e., be compatible with) world state $\mathcal{I}$ of Eq. (2) is

$$
\begin{aligned}
s^{\mathcal{I}} & =\min _{\ell \in \mathcal{L}} \max \left\{1-s(\ell), \min \left\{s(\ell), \ell^{\mathcal{I}}\right\}\right\} \\
& =\min \left\{\operatorname { m a x } \left\{1-s(c), \min \left\{s(c), c^{\mathcal{I}}\right\}, \max \left\{1-s(p), \min \left\{s(p), p^{\mathcal{I}}\right\}, \max \left\{1-s(\neg p), \min \left\{s(\neg p), 1-p^{\mathcal{I}}\right\}\right\}\right.\right.\right. \\
& =\min \{\max \{1-1, \min \{1,1\}, \max \{1-0.5, \min \{0.5,0.2\}, \max \{1-1, \min \{1,0.8\}\}=0.5 .
\end{aligned}
$$

Examples of fuzzy state descriptions that totally describe $\mathcal{I}$ are

$$
\emptyset, \quad \frac{1}{c}, \quad \frac{1}{c}+\frac{0.8}{f}, \quad \frac{0.8}{f}+\frac{0.2}{\neg f}, \quad \frac{1}{\neg g}+\frac{0.2}{p}+\frac{1}{\neg y}, \ldots .
$$

Proposition 1. Let $\mathcal{I}$ be a fuzzy interpretation; for all $s \in \mathcal{S}$,

$$
\begin{aligned}
s^{\mathcal{I}}= & \min _{p \in \mathcal{A}}\left\{\max \{1-s(p), s(p)\}, \max \left\{1-s(p), p^{\mathcal{I}}\right\},\right. \\
& \left.\max \{1-s(\neg p), s(\neg p)\}, \max \left\{1-s(\neg p), 1-p^{\mathcal{I}}\right\}\right\} .
\end{aligned}
$$

Proof. By Definition 2,

$$
\begin{aligned}
s^{\mathcal{I}}= & \min _{\ell \in \mathcal{L}} \max \left\{1-s(\ell), \min \left\{s(\ell), \ell^{\mathcal{I}}\right\}\right\} \\
= & \min _{p \in \mathcal{A}}\left\{\max \left\{1-s(p), \min \left\{s(p), p^{\mathcal{I}}\right\}\right\}, \max \left\{1-s(\neg p), \min \left\{s(\neg p),(\neg p)^{\mathcal{I}}\right\}\right\}\right\} \\
= & \min _{p \in \mathcal{A}}\left\{\max \left\{1-s(p), \min \left\{s(p), p^{\mathcal{I}}\right\}\right\}, \max \left\{1-s(\neg p), \min \left\{s(\neg p), 1-p^{\mathcal{I}}\right\}\right\}\right\} \\
= & \min _{p \in \mathcal{A}}\left\{\max \{1-s(p), s(p)\}, \max \left\{1-s(p), p^{\mathcal{I}}\right\},\right. \\
& \left.\quad \max \{1-s(\neg p), s(\neg p)\}, \max \left\{1-s(\neg p), 1-p^{\mathcal{I}}\right\}\right\} .
\end{aligned}
$$

Proposition 2. Let s be a fuzzy state description and $\mathcal{I}$ a fuzzy interpretation: then,

$$
s^{\mathcal{I}}=1 \Rightarrow \forall p \in \mathcal{A}, s(p)=p^{\mathcal{I}} .
$$


Proof. Since $(A \supset B) \equiv(\neg B \subset \neg A)$, instead of proving the thesis directly, we prove its equivalent $\exists p \in$ $\mathcal{A}, \quad s(p) \neq p^{\mathcal{I}} \Rightarrow s^{\mathcal{I}} \neq 1$. Let us assume $p$ is an atom such that $s(p) \neq p^{\mathcal{I}}$; then $\max \left\{1-s(p), p^{\mathcal{I}}\right\} \neq 1$. Therefore, because of Proposition $1, s^{\mathcal{I}} \neq 1$.

Proposition 3. Let s be a fuzzy state description and $\mathcal{I}$ a fuzzy interpretation: then,

$$
\forall p \in \mathcal{A}, s(p)=p^{\mathcal{I}} \Rightarrow s^{\mathcal{I}}=\min _{p \in \mathcal{A}} \max \left\{p^{\mathcal{I}}, 1-p^{\mathcal{I}}\right\}
$$

Proof. The thesis follows trivially by replacing $s(p)$ by $p^{\mathcal{I}}$ in Proposition 1 .

Corollary 4. Let s be a fuzzy state description and $\mathcal{I}$ a crisp interpretation: then,

$$
\forall p \in \mathcal{A}, s(p)=p^{\mathcal{I}} \Rightarrow s^{\mathcal{I}}=1
$$

Proof. The thesis is a direct consequence of Proposition 3, when $\mathcal{I}$ is crisp, i.e., when, for all $p \in \mathcal{A}, p^{\mathcal{I}} \in\{0,1\}$ and, therefore, $\max \left\{p^{\mathcal{I}}, 1-p^{\mathcal{I}}\right\}=1$.

\subsection{Fuzzy entailment of expressions}

An expression is a well-formed formula in propositional logic employing literals defined in the problem domain. The degree to which a given world state $\mathcal{I}$ satisfies an expression $\varepsilon$, i.e., its truth degree in $\mathcal{I}$, is denoted $\varepsilon^{\mathcal{I}}$.

Definition 3. A state description $s$ entails an expression $\varepsilon$, denoted $s \vDash \varepsilon$, if and only if, for all world state $\mathcal{I}, s^{\mathcal{I}} \leqslant \varepsilon^{\mathcal{I}}$.

The $\vDash$ relation is a crisp one. It is useful to define a fuzzy entailment relation as well, on the basis of the Łukasiewicz implication as suggested in [6].

Definition 4. The degree to which a state description $s$ entails an expression $\varepsilon$, is

$$
\operatorname{Ent}(s, \varepsilon)=\inf _{\mathcal{I}} \min \left\{1,1-s^{\mathcal{I}}+\varepsilon^{\mathcal{I}}\right\}
$$

It is easy to verify that, if $s \models \varepsilon, \operatorname{Ent}(s, \varepsilon)=1$, because, by Definition $3, \varepsilon^{\mathcal{I}}-s^{\mathcal{I}} \geqslant 0$ for all $\mathcal{I}$, therefore $1-s^{\mathcal{I}}+\varepsilon^{\mathcal{I}} \geqslant 1$, and

$$
\operatorname{Ent}(s, \varepsilon)=\inf _{\mathcal{I}} \min \left\{1,1-s^{\mathcal{I}}+\varepsilon^{\mathcal{I}}\right\}=\inf _{\mathcal{I}} 1=1
$$

This fuzzy entailment relation may be used to define the fuzzy sets of all the state descriptions that entail a given expression.

Definition 5. The fuzzy set of all the state descriptions that entail an expression $\varepsilon$, is $S_{\varepsilon}$. The degree to which a state description $s$ belongs to $S_{\varepsilon}$ is

$$
S_{\varepsilon}(s)=\operatorname{Ent}(s, \varepsilon)
$$

\section{Fuzzy possibilistic actions}

Now that we know how to represent actual or possible world states, as well as what a planning agent knows about them-fuzzy state descriptions, it is time to account for the uncertainty about the actual world state at a given point of a plan. Based on our assumptions, the source of uncertainty is nondeterminism of actions. Therefore, we now turn to 
defining the syntax and semantics of possibilistic actions in the framework of the formalism introduced in the previous section.

\subsection{Representing uncertainty about action results}

We represent uncertainty about the world state by means of possibility distributions $\pi: \mathcal{S} \rightarrow[0,1]$.

A possibility distribution $\pi$ on $\mathcal{S}$ induces a possibility measure $\Pi: 2^{\mathcal{S}} \rightarrow[0,1]$ and a necessity measure $N: 2^{\mathcal{S}} \rightarrow$ $[0,1]$, defined, for all crisp set of state descriptions $X \subseteq \mathcal{S}$, as

$$
\begin{gathered}
\Pi(X)=\sup _{s \in X} \pi(s), \\
N(X)=1-\Pi(\bar{X})=\inf _{s \notin X}(1-\pi(s)) .
\end{gathered}
$$

These measures can be extended to fuzzy sets of state descriptions as follows: for all fuzzy set of state descriptions $X: \mathcal{S} \rightarrow[0,1]$

$$
\begin{gathered}
\Pi(X)=\sup _{s \in \mathcal{S}} \min \{X(s), \pi(s)\}, \\
N(X)=1-\Pi(\bar{X})=\inf _{s \in \mathcal{S}} \max \{X(s), 1-\pi(s)\} .
\end{gathered}
$$

\subsection{Representing actions}

Definition 6. An action $a$ with possibilistic effects is a set of possibilistic effects, i.e., $a=\left\{p e_{1}, \ldots, p e_{m}\right\}$, where

$$
p e_{i}=\left\langle\text { Context }_{i},\left(\pi_{i 1}, \operatorname{Eff}_{i 1}\right), \ldots,\left(\pi_{i n_{i}}, \operatorname{Eff}_{i n_{i}}\right)\right\rangle,
$$

where, for all $i$ and $j$,

- Context $t_{i}$ is the context that triggers the $i$ th possibilistic effect;

- $\pi_{i j} \in(0,1]$ is the degree of possibility of the relevant effect and, for all $i$, there exists a $j$ such that $\pi_{i j}=1$;

- Eff $_{i j}=\left(A_{i j}, R_{i j}\right), A_{i j} \cap R_{i j}=\emptyset$, where $A_{i j}$ is a (crisp) set of literals that must be asserted (i.e., added to the state description) and $R_{i j}$ is a (crisp) set of literals that must be retracted (i.e., deleted from the state description).

Furthermore, the contexts of an action must be such that:

(1) for all $s \in \mathcal{S}$, and for all interpretations $\mathcal{I}$, there exists an $i$ such that $\operatorname{Context}_{i}^{\mathcal{I}} \geqslant s^{\mathcal{I}}$.

(2) the contexts are totally overlapping, i.e., $\sum_{i}$ Context $_{i}=1$.

In our example, we might define the following four possibilistic actions:

- sow standard-quality seeds (sowStd);

- sow resistant seeds (sowRes);

- treat the crop with pesticides (treat);

- harvest the crop (harvest).

These four actions would be formally defined as follows:

$$
\begin{aligned}
\text { sowStd } & =\{\langle f,(1,\{c, g\},\{\neg c\}),(0.2,\{c, \neg g\},\{\neg c\})\rangle,\langle\neg f,(0.7,\{c, g\},\{\neg c\}),(1,\{c, \neg g\},\{\neg c\})\rangle\}, \\
\text { sowRes } & =\{\langle f,(1,\{c, g\},\{\neg c\}),(0.1,\{c, \neg g\},\{\neg c\})\rangle,\langle\neg f,(1,\{c, g\},\{\neg c\}),(0.5,\{c, \neg g\},\{\neg c\})\rangle\}, \\
\text { treat } & =\{\langle p,(1,\{\neg p\},\{p\}),(0.1, \emptyset, \emptyset)\rangle,\langle\neg p,(1, \emptyset, \emptyset)\rangle\}, \\
\text { harvest } & =\{\langle s \wedge g \wedge \neg p,(1,\{y\}, \emptyset)\rangle,\langle\neg s \vee \neg g \vee p,(1,\{\neg y\}, \emptyset)\rangle\} .
\end{aligned}
$$




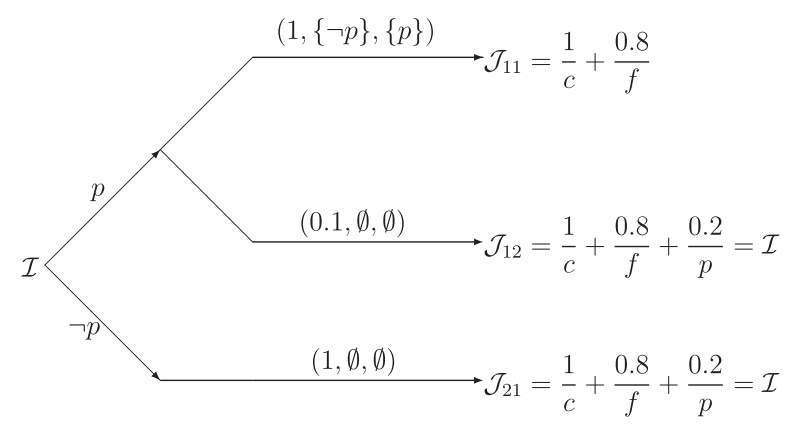

Fig. 1. A diagram illustrating the results of applying action treat to world state $\mathcal{I}$.

The semantics of possibilistic actions can be worked out by regarding a possibilistic action as a set of fuzzy IF-THEN rules of the form

IF Context $_{1}$ THEN Eff $_{11}$ with possibility $\pi_{11}$;

:

IF Context $_{1}$ THEN Eff ${ }_{1 n_{1}}$ with possibility $\pi_{1 n_{1}}$;

$\vdots$

IF Context THEN Eff $_{m 1}$ with possibility $\pi_{m 1}$;

$\vdots$

IF Context THEN Eff $_{m n_{1}}$ with possibility $\pi_{m n_{m}}$

The above rules are to be interpreted as if they were in a logical disjunction with one another [29]; as a consequence, the overall effect of a possibilistic action should be construed as the fuzzy union of the effect of each rule, with a degree given by the degree of satisfaction of the relevant IF part and the possibility of the effect.

\subsection{Action semantics}

The semantics of a possibilistic action is established by the following definition.

Definition 7. Let $\mathcal{I}$ be a world state (i.e., a fuzzy interpretation), and $a=\left\{\left\langle\operatorname{Context}_{i},\left(\pi_{i 1}, \operatorname{Eff}_{i 1}\right), \ldots,\left(\pi_{i n_{i}}\right.\right.\right.$, Eff $\left.\left.\left._{i n_{i}}\right)\right\rangle,\right\}_{i=1, \ldots, m}$ be a possibilistic action. The world state resulting from possibilistic effect Eff $i_{i j}$ is a fuzzy interpretation $\mathcal{J}_{i j}$ such that, for all literal $\ell \in \mathcal{L}$,

(1) $\ell^{\mathcal{J}_{i j}}=\ell^{\mathcal{I}}$, if $\ell \notin \operatorname{Eff}_{i j}$ (a sort of law of inertia);

(2) $\ell^{\mathcal{J}_{i j}}=\max \left\{\ell^{\mathcal{I}}, \pi_{i j}\right\}$, if $\ell \in A_{i j}$;

(3) $\ell^{\mathcal{J}_{i j}}=\min \left\{\ell^{\mathcal{I}}, 1-\pi_{i j}\right\}$, if $\ell \in R_{i j}$.

The possibility for world state $\mathcal{I}^{\prime}$ of resulting from execution of action $a$ in $\mathcal{I}$ is

$$
\pi\left(\mathcal{I}^{\prime} \mid \mathcal{I}, a\right)= \begin{cases}\max _{i, j: \mathcal{J}_{i j}=\mathcal{I}^{\prime}} \min \left\{\text { Context }_{i}^{\mathcal{I}}, \pi_{i j}\right\} & \text { if } \exists i, j: \mathcal{J}_{i j}=\mathcal{I}^{\prime} \\ 0 & \text { otherwise. }\end{cases}
$$

The diagram in Fig. 1 illustrates what applying action treat to world state $\mathcal{I}$ of Eq. (2) would mean: the first context, $p$, is satisfied with degree 0.2 in $\mathcal{I}$, whereas the second context, $\neg p$, is satisfied with degree $0.8 . A_{11}=\{\neg p\}$ and $R_{11}=\{p\}$, so that

$$
\begin{aligned}
(\neg p)^{\mathcal{J}_{11}} & =\max \left\{(\neg p)^{\mathcal{I}}, \pi_{11}\right\}=\max \{0.8,1\}=1, \\
p^{\mathcal{J}_{11}} & =\min \left\{p^{\mathcal{I}}, 1-\pi_{11}\right\}=\min \{0.2,0\}=0 .
\end{aligned}
$$

The other two effects of treat leave the state of affairs unchanged. 
Intuitively, treating the field when crop has been sown, the weather conditions are quite favorable, and the crop is little affected by pest, will result in a situation where the crop is totally unaffected $\left(\mathcal{I}_{11}\right)$, if the treatment is successful, or in a situation where nothing has changed $\left(\mathcal{I}_{12}=\mathcal{I}\right)$, if the treatment is unsuccessful. The way it is written, the action says even something more: to the extent that the crop is unaffected by pest, which in this case is 0.8 , treating it will make no difference, and the crop will continue to be little affected $\left(\mathcal{I}_{21}=\mathcal{I}\right)$.

We can thus evaluate the possibilities of the two outcomes of treating the field:

$$
\begin{aligned}
\pi\left(\mathcal{J}_{11} \mid \mathcal{I}, \text { treat }\right) & =\min \left\{p^{\mathcal{I}}, \pi_{11}\right\}=\min \{0.2,1\}=0.2, \\
\pi(\mathcal{I} \mid \mathcal{I}, \text { treat }) & =\max \left\{\min \left\{p^{\mathcal{I}}, \pi_{12}\right\}, \min \left\{(\neg p)^{\mathcal{I}}, \pi_{21}\right\}\right\} \\
& =\max \{\min \{0.2,0.1\}, \min \{0.8,1\}\}=\max \{0.1,0.8\}=0.8 .
\end{aligned}
$$

The next definition describes the way a state description should be updated to reflect changes in the world state resulting from the execution of a possibilistic action.

Definition 8. Let $a$ be a possibilistic action, $s$ a fuzzy state description, and $\mathcal{I}$ an actual world state. The result of executing $a$ on $s$ in $\mathcal{I}$ is a new fuzzy state description $s^{\prime}=a^{\mathcal{I}}(s)$, such that, for all $\ell \in \mathcal{L}$,

$$
s^{\prime}(\ell)=\max _{i, j} \min \left\{\max \left\{s(\ell), A_{i j}^{\mathcal{I}}(\ell)\right\}, 1-R_{i j}^{\mathcal{I}}(\ell)\right\},
$$

where

$$
\begin{aligned}
& A_{i j}^{\mathcal{I}}(\ell)=\min \left\{\text { Context }_{i}^{\mathcal{I}}, \pi_{i j}, A_{i j}(\ell)\right\}, \\
& R_{i j}^{\mathcal{I}}(\ell)=\min \left\{\text { Context }_{i}^{\mathcal{I}}, \pi_{i j}, R_{i j}(\ell)\right\} .
\end{aligned}
$$

Of course, the planner cannot know what the actual state of the world corresponding to $s$ could be; in fact, in principle, it should take all world states $\mathcal{I}$ into account with the degree to which they are represented by $s$ in order to evaluate the possible outcome of action $a$.

In order to illustrate Definition 8, let us assume action treat is applied on fuzzy state description $s$ of Eq. (12) when the actual state of the world is $\mathcal{I}$ of Eq. (2). A new state description $s^{\prime}$ would ensue, such that

$$
s^{\prime}(p)=\max _{i j} \min \left\{\max \left\{0.5, A_{i j}^{\mathcal{I}}(p)\right\}, 1-R_{i j}^{\mathcal{I}}(p)\right\} .
$$

Now,

$$
\begin{array}{ll}
A_{11}^{\mathcal{I}}(p)=0, & R_{11}^{\mathcal{I}}(p)=0.2 \\
A_{12}^{\mathcal{I}}(p)=0, & R_{12}^{\mathcal{I}}(p)=0, \\
A_{21}^{\mathcal{I}}(p)=0, & R_{21}^{\mathcal{I}}(p)=0
\end{array}
$$

therefore,

$$
s^{\prime}(p)=\max \{\min \{\max \{0.5,0\}, 0.8\}=0.5, \min \{\max \{0.5,0\}, 1\}=0.5, \min \{\max \{0.5,0\}, 1\}=0.5\}=0.5 .
$$

Similarly, we can calculate $s^{\prime}(\neg p)=1$. Therefore, since it can be easily verified that, for all literal $\ell$ which does neither occur in $A_{i j}$ nor in $R_{i j}, s^{\prime}(\ell)=s(\ell), s^{\prime}=1 / s+0.5 / p+1 / \neg p=s$, and we can conclude that, in $\mathcal{I}$, if treat is applied on $s, s$ does not change, even though, of course, $\mathcal{I}$ might change.

\subsection{Uncertain outcome of action execution}

We now turn to characterizing the uncertainty of the outcome of executing an action from the point of view of what the planning agent knows, i.e., of fuzzy state descriptions.

Definition 9. The (crisp) set of all world states (i.e., interpretations) that can result from the execution of possibilistic action $a$ in world state $\mathcal{I}$ is

$$
\operatorname{Res}(\mathcal{I}, a)=\left\{\mathcal{I}^{\prime}: \pi\left(\mathcal{I}^{\prime} \mid \mathcal{I}, a\right)>0\right\}
$$


Definition 10. The (crisp) set of all state descriptions that can result from the execution of a possibilistic action $a$ given a state description $s$ is

$$
\operatorname{Res}(s, a)=\left\{s^{\prime}: \exists \mathcal{I}: s^{\prime}=a^{\mathcal{I}}(s)\right\} .
$$

Definition 11. The possibility that state description $s^{\prime}$ describes the world state resulting from executing possibilistic action $a$ in world state $\mathcal{I}$ is

$$
\pi\left(s^{\prime} \mid \mathcal{I}, a\right)=\sup _{\mathcal{I}^{\prime}} \pi\left(\mathcal{I}^{\prime} \mid \mathcal{I}, a\right)
$$

Definition 12. The possibility that state description $s^{\prime}$ is the result of executing a possibilistic action $a$ given the state description $s$ is

$$
\pi\left(s^{\prime} \mid s, a\right)=\sup _{\mathcal{I}} \min \left\{\pi\left(s^{\prime} \mid \mathcal{I}, a\right), s^{\mathcal{I}}\right\}
$$

Proposition 5. Let $s$ and $s^{\prime}$ be state descriptions, and a be a possibilistic action:

$$
\pi\left(s^{\prime} \mid s, a\right)=\sup _{\mathcal{I}} \max _{i j} \min \left\{\text { Context }_{i}^{\mathcal{I}}, \pi_{i j}, s^{\mathcal{I}}\right\} .
$$

Proof. By Definition 5,

$$
\begin{aligned}
\pi\left(s^{\prime} \mid s, a\right) & =\sup _{\mathcal{I}} \min \left\{\pi\left(s^{\prime} \mid \mathcal{I}, a\right), s^{\mathcal{I}}\right\} \\
& =\sup _{\mathcal{I}} \min \left\{\sup _{\mathcal{I}^{\prime}} \pi\left(\mathcal{I}^{\prime} \mid \mathcal{I}, a\right), s^{\mathcal{I}}\right\} \\
& =\sup _{\mathcal{I}} \min \left\{\sup _{\mathcal{I}^{\prime}} \max _{i, j: \mathcal{J}_{i j}=\mathcal{I}^{\prime}} \min \left\{\text { Context }_{i}^{\mathcal{I}}, \pi_{i j}\right\}, s^{\mathcal{I}}\right\} \\
& =\sup _{\mathcal{I}} \sup _{\mathcal{I}^{\prime}} \max _{i, j: \mathcal{J}_{i j}=\mathcal{I}^{\prime}} \min \left\{\text { Context }_{i}^{\mathcal{I}}, \pi_{i j}\right\}, s^{\mathcal{I}} \\
& =\sup _{\mathcal{I}} \max _{i j} \min \left\{\operatorname{Context}_{i}^{\mathcal{I}}, \pi_{i j}, s^{\mathcal{I}}\right\} .
\end{aligned}
$$

To continue with the previous example, let us now assume

$$
s=\frac{1}{s}+\frac{0.2}{p},
$$

and that applying action treat on $s$ in $\mathcal{I}$ yields

$$
s^{\prime}=\frac{1}{s}+\frac{0.2}{p}+\frac{0.2}{\neg p} .
$$

We want to calculate the possibility that $s^{\prime}$ be the outcome of applying action treat on $s$,

$$
\pi\left(s^{\prime} \mid s, \text { treat }\right)=\sup _{\mathcal{I}} \min \left\{\pi\left(s^{\prime} \mid \mathcal{I}, \text { treat }\right), s^{\mathcal{I}}\right\} .
$$

Therefore, $\pi\left(s^{\prime} \mid s\right.$, treat $) \geqslant \min \left\{\pi\left(s^{\prime} \mid \mathcal{I}\right.\right.$, treat $\left.), 1\right\}=\pi\left(s^{\prime} \mid \mathcal{I}\right.$, treat $)$, now,

$$
\begin{aligned}
\pi\left(s^{\prime} \mid \mathcal{I}, \text { treat }\right) & =\sup _{\mathcal{I}^{\prime}} \min \left\{\pi\left(\mathcal{I}^{\prime} \mid \mathcal{I}, \text { treat }\right), s^{\prime \mathcal{I}^{\prime}}\right\} \\
& =\max \left\{\min \left\{0.2, s^{\prime} \mathcal{J}_{11}\right\}, \min \left\{0.8, s^{\prime \mathcal{I}}\right\}\right\} \\
& =\max \{\min \{0.2,0.8\}, \min \{0.8,0.8\}\}=0.8 .
\end{aligned}
$$




\subsection{Reasoning about actions}

The two main reasoning tasks on actions have to do with answering the questions:

- What would be the consequence of executing a given action?

- Can a given action be executed in a given (partially known) world state?

Answering the former question requires being able to determine whether a given condition (i.e., an expression) will hold after the action is executed. Such a reasoning task is usually called projection. The reasoning task implied by answering the latter question is called executability.

Definition 13 (Projection). The degree to which an expression $\varepsilon$ is a consequence (i.e., a projection) of applying a possibilistic action $a$ given a state description $s$ is

$$
\pi(\varepsilon \mid s, a)=\sup _{s^{\prime} \in \operatorname{Res}(s, a)} \min \left\{\pi\left(s^{\prime} \mid s, a\right), \operatorname{Ent}\left(s^{\prime}, \varepsilon\right)\right\} .
$$

Definition 14 (Executability). The degree to which a possibilistic action $a$ is executable given a state description $s$ is

$$
\eta(s, a)=\sup _{\mathcal{I}} \min \left\{s^{\mathcal{I}}, \max _{j} \operatorname{Context}_{j}^{\mathcal{I}}\right\} .
$$

Definition 15. The degree to which a possibilistic action $a$ is executable given a possibility distribution $\pi$ of state descriptions is

$$
\eta(\pi, a)=\sup _{s} \min \{\pi(s), \eta(s, a)\} .
$$

\section{Action plans}

The next step is to chain actions, thus obtaining trajectories in the space of state descriptions, and action plans.

Definition 16. The degree to which a sequence $\left\langle a_{i}\right\rangle_{i=0}^{N-1}$ of possibilistic actions is executable given an initial state description $s_{0}$ is given by

$$
\eta\left(s_{0},\left\langle a_{i}\right\rangle_{i=0}^{N-1}\right)=\min \left\{\begin{array}{l}
\eta\left(s_{0}, a_{0}\right), \\
N-1 \\
\min _{i=1} \sup _{i} \min \left\{\pi\left(s_{i} \mid s_{i-1}, a_{i-1}\right), \eta\left(s_{i}, a_{i}\right)\right\}
\end{array}\right\} .
$$

Definition 17 (Trajectory). A trajectory is a sequence $\tau=\left\langle s_{0}, \ldots s_{N}\right\rangle$ of state descriptions.

We classically define a plan of actions as a set of ordered actions.

Definition 18 (Action plan). A sequential plan $P_{\text {seq }}$ is a totally ordered set of actions $\left\langle a_{i}\right\rangle_{i=0}^{N-1}$.

To execute a plan $P=\left\langle a_{i}\right\rangle_{i=0}^{N-1}$ is to execute in sequence $a_{0}, a_{1}, \ldots, a_{N-1}$. The strong assumption of nonobservability underlies the way the execution is monitored. Indeed we suppose that despite the uncertainty concerning the effects of the actions, a plan is executed blindly, without any information gathering between steps. The following proposition relies on this assumption.

Proposition 6. The possibility to reach a world state that satisfies a given state description $s_{N}$, starting from a world state described by $s_{0}$ by executing a sequence $\left\langle a_{i}\right\rangle_{i=0}^{N-1}$ of possibilistic actions is given by

$$
\pi\left(s_{N} \mid s_{0},\left\langle a_{i}\right\rangle_{i=0}^{N-1}\right)=\sup _{\tau=\left\langle s_{0}, \ldots s_{N}\right\rangle} \min _{i=0}^{N-1} \pi\left(s_{i+1} \mid s_{i}, a_{i}\right) .
$$


Proof.

$$
\begin{aligned}
\pi\left(s_{N} \mid s_{0},\left\langle a_{i}\right\rangle_{i=0}^{N-1}\right)= & \sup _{s_{1}} \min \left\{\pi\left(s_{1} \mid s_{0}, a_{0}\right), \pi\left(s_{N} \mid s_{1},\left\langle a_{i}\right\rangle_{i=1}^{N-1}\right)\right\} \\
= & \sup _{s_{1}} \min \left\{\pi\left(s_{1} \mid s_{0}, a_{0}\right), \sup _{s_{2}} \min \left\{\pi\left(s_{2} \mid s_{1}, a_{1}\right), \pi\left(s_{N} \mid s_{2},\left\langle a_{i}\right\rangle_{i=2}^{N-1}\right)\right\}\right\} \\
= & \sup _{s_{1}} \sup _{s_{2}} \min \left\{\pi\left(s_{1} \mid s_{0}, a_{0}\right), \pi\left(s_{2} \mid s_{1}, a_{1}\right), \pi\left(s_{N} \mid s_{2},\left\langle a_{i}\right\rangle_{i=2}^{N-1}\right)\right\} \\
& \vdots \\
= & \sup _{s_{1}} \ldots \sup _{s_{N}} \min \left\{\pi\left(s_{1} \mid s_{0}, a_{0}\right), \ldots, \pi\left(s_{N} \mid s_{N-1}, a_{N-1}\right)\right\} \\
= & \sup _{\tau} \min _{i=0}^{N-1} \pi\left(s_{i+1} \mid s_{i}, a_{i}\right) .
\end{aligned}
$$

Proposition 7. The possibility to reach a world state that satisfies a given expression $\varepsilon$, starting from a world state described by $s_{0}$ by executing a sequence $\left\langle a_{i}\right\rangle_{i=0}^{N-1}$ of possibilistic actions is given by

$$
\pi\left(\varepsilon \mid s_{0},\left\langle a_{i}\right\rangle_{i=0}^{N-1}\right)=\sup _{s_{N}} \min \left\{\pi\left(s_{N} \mid s_{0},\left\langle a_{i}\right\rangle_{i=0}^{N-1}\right), \operatorname{Ent}\left(s_{N}, \varepsilon\right)\right\} .
$$

Definition 19. The necessity of reaching a world state that satisfies a given expression $\varepsilon$, starting from a world state described by $s_{0}$ by executing a sequence $\left\langle a_{i}\right\rangle_{i=0}^{N-1}$ of possibilistic actions is given by

$$
v\left(\varepsilon \mid s_{0},\left\langle a_{i}\right\rangle_{i=0}^{N-1}\right)=1-\pi\left(\neg \varepsilon \mid s_{0},\left\langle a_{i}\right\rangle_{i=0}^{N-1}\right) .
$$

\section{Fuzzy possibilistic planning problems}

We now have all the elements needed to represent a planning problem within the framework of the proposed formalism. We will then characterize solutions to such a planning problem, by introducing the notion of a $\gamma$-acceptable plan, i.e., a plan whose execution is guaranteed to lead to a goal state with a degree of necessity $\gamma$, and by showing that, under certain conditions, finding a solution to such planning problem is no harder than solving a related planning problem with crisp and complete information.

\subsection{Representing problems}

Definition 20 (Fuzzy possibilistic planning problem). A possibilistic planning problem is a triple $\Delta=\left\langle s_{0}, \varepsilon_{G}, A\right\rangle$, where $s_{0}$ is a description of the initial state, $\varepsilon_{G}$ is an expression defining the set of goal states $\left(G=\mathcal{S}\left(\varepsilon_{G}\right)\right)$, and $A$ is the set of available actions.

\subsection{Solution plans and $\gamma$-acceptability}

Definition 21 (Solution plan). Let $\Delta$ be a fuzzy possibilistic planning problem and $P$ a sequential plan. $P$ is said to be:

- $\gamma$-acceptable, if $N\left[G \mid s_{0}, P\right] \geqslant \gamma$;

- a maximal-certainty plan, if $P$ is $\gamma$-acceptable and $\forall P^{\prime}$ such that $P^{\prime}$ is $\gamma^{\prime}$-acceptable, $\gamma^{\prime} \leqslant \gamma$.

Proposition 8. Let $P=\left\langle a_{i}\right\rangle_{i=0}^{N-1}$ be a $\gamma$-acceptable sequential plan for a fuzzy possibilistic planning problem $\Delta=\left\langle s_{0}, \varepsilon_{G}, A\right\rangle$.

For all trajectory $\left\langle s_{0}, \ldots, s_{N}\right\rangle$, a sufficient condition for $s_{N}$ to be a goal state with degree at least $\gamma$, in symbols $G\left(s_{N}\right) \geqslant \gamma$, is that, for $i=0, \ldots, N-1$,

$$
\pi\left(s_{i+1} \mid s_{i}, a_{i}\right)>1-\gamma .
$$


Proof. $P$ is $\gamma$-acceptable, therefore, $N\left[G \mid s_{0}, P\right]=N\left[G \mid s_{0},\left\langle a_{i}\right\rangle_{i=0}^{N-1}\right] \geqslant \gamma$. By Eq. (22),

$$
N\left[G \mid s_{0},\left\langle a_{i}\right\rangle_{i=0}^{N-1}\right]=\inf _{s_{N}} \max \left\{G\left(s_{N}\right), 1-\pi\left(s_{N} \mid s_{0},\left\langle a_{i}\right\rangle_{i=0}^{N-1}\right)\right\} .
$$

Therefore,

$$
\begin{aligned}
& N\left[G \mid s_{0}, P\right] \geqslant \gamma \\
& \Leftrightarrow \inf _{s_{N}} \max \left\{G\left(s_{N}\right), 1-\pi\left(s_{N} \mid s_{0},\left\langle a_{i}\right\rangle_{i=0}^{N-1}\right)\right\} \geqslant \gamma \\
& \Leftrightarrow \forall s_{N} \max \left\{G\left(s_{N}\right), 1-\pi\left(s_{N} \mid s_{0},\left\langle a_{i}\right\rangle_{i=0}^{N-1}\right)\right\} \geqslant \gamma \\
& \Leftrightarrow \forall s_{N}\left(G\left(s_{N}\right)<\gamma \Rightarrow \pi\left(s_{N} \mid s_{0},\left\langle a_{i}\right\rangle_{i=0}^{N-1}\right) \leqslant 1-\gamma\right) \\
& \Leftrightarrow \forall s_{N}\left(\pi\left(s_{N} \mid s_{0},\left\langle a_{i}\right\rangle_{i=0}^{N-1}\right)>1-\gamma \Rightarrow G\left(s_{N}\right) \geqslant \gamma\right) \\
& \Leftrightarrow \forall s_{N}\left(\sup _{\left\langle s_{0}, \ldots s_{N}\right\rangle} \min _{i=0, \ldots, N-1} \pi\left(s_{i+1} \mid s_{i}, a_{i}\right)>1-\gamma \Rightarrow G\left(s_{N}\right) \geqslant \gamma\right) \\
& \Leftrightarrow \forall s_{N}\left(\exists\left\langle s_{0}, \ldots s_{N}\right\rangle \forall i\left(\pi\left(s_{i+1} \mid s_{i}, a_{i}\right)>1-\gamma\right) \Rightarrow G\left(s_{N}\right) \geqslant \gamma\right) \\
& \Rightarrow \forall\left\langle s_{0}, \ldots s_{N}\right\rangle\left(\forall i\left(\pi\left(s_{i+1} \mid s_{i}, a_{i}\right)>1-\gamma\right) \Rightarrow G\left(s_{N}\right) \geqslant \gamma\right) \text {, }
\end{aligned}
$$

which is the thesis.

The previous propositions give us a sufficient condition for a $\gamma$-acceptable plan to be able to solve a planning problem with a necessity at least $\gamma$. The next question is when such a sufficient condition is guaranteed to hold. Any answer to such question would give us a class of fuzzy possibilistic planning problems that are "less hard" to solve. The following proposition identifies one such class.

Proposition 9. Let $\Delta=\left\langle s_{0}, \varepsilon_{G}\right.$, A $\rangle$ be a fuzzy possibilistic planning problem such that

(1) there exists an interpretation $\mathcal{I}_{0}$ such that $s_{0}^{\mathcal{I}_{0}}>1-\gamma$;

(2) for all $a \in A$, every possibilistic effect pe ${ }_{j}$ of a contains only one effect Eff $_{j}$, whose possibility is 1;

(3) for all $a \in A$, if a contains a possibilistic effect pe $e_{j}$ such that $R_{j} \neq \emptyset$, then $j=1$ and a contains no other effect. ${ }^{1}$

If $P=\left\langle a_{i}\right\rangle_{i=0}^{N-1}$ is a $\gamma$-acceptable sequential plan for problem $\Delta$, there exists a trajectory $\left\langle s_{0}, \ldots, s_{N}\right\rangle$ obtained by executing $P$, such that $G\left(s_{N}\right) \geqslant \gamma$.

Proof. We begin by proving that, given $P$, there exists a trajectory $\left\langle s_{0}, \ldots, s_{N}\right\rangle$ such that, for all $i=0, \ldots, N-1$, $\pi\left(s_{i+1} \mid s_{i}, a_{i}\right)>1-\gamma$.

For all actions $a_{i}, i=0, \ldots, N-1$, given state description $s_{i}$, by definition of a possibilistic action, for all interpretation $\mathcal{I}_{i}$ there must be a context Context ${ }_{j i}$ such that Context ${ }_{j_{i}}^{\mathcal{I}_{i}} \geqslant s_{i}^{\mathcal{I}_{i}}$. Now, for a given interpretation $\mathcal{I}_{i}$,

$$
\begin{aligned}
\pi\left(s_{i+1} \mid s_{i}, a_{i}\right) & =\sup _{\mathcal{I}} \max _{j k} \min \left\{\operatorname{Context}_{j}^{\mathcal{I}}, \pi_{j k}, s_{i}^{\mathcal{I}}\right\} \\
& =\sup _{\mathcal{I}} \max _{j} \min \left\{\operatorname{Context}_{j}^{\mathcal{I}}, s_{i}^{\mathcal{I}}\right\} \quad\left(\text { because } \forall j \max _{k} \pi_{j k}=1\right) \\
& \geqslant \sup _{\mathcal{I}} \min \left\{\operatorname{Context}_{j_{i}}^{\mathcal{I}}, s_{i}^{\mathcal{I}}\right\} \\
& \geqslant \min \left\{\operatorname{Context}_{j_{i}}^{\mathcal{I}_{i}}, s_{i}^{\mathcal{I}_{i}}\right\}=s_{i}^{\mathcal{I}_{i}} .
\end{aligned}
$$

Therefore, $\pi\left(s_{i+1} \mid s_{i}, a_{i}\right)>1-\gamma$ if there exists an interpretation $\mathcal{I}_{i}$ such that $s_{i}^{\mathcal{I}_{i}}>1-\gamma$.

By hypothesis, there exists an interpretation $\mathcal{I}_{0}$ such that $s_{0}^{\mathcal{I}_{0}}>1-\gamma$. We now prove by induction that, if there exists an interpretation $\mathcal{I}_{i}$ such that $s_{i}^{\mathcal{I}_{i}}>1-\gamma$, then there exist an interpretation $\mathcal{I}_{i+1} \in \operatorname{Res}\left(\mathcal{I}_{i}, a_{i}\right)$ such that $s_{i+1}^{\mathcal{I}_{i+1}}>$ $1-\gamma$.

\footnotetext{
${ }^{1}$ Essentially, this condition requires that all actions that retract some literal must be unconditional; indeed, by Definition 6 , it must be Context $1=\top$.
} 
Table 1

Synopsis of the cases considered in the induction step of the proof of Proposition 9

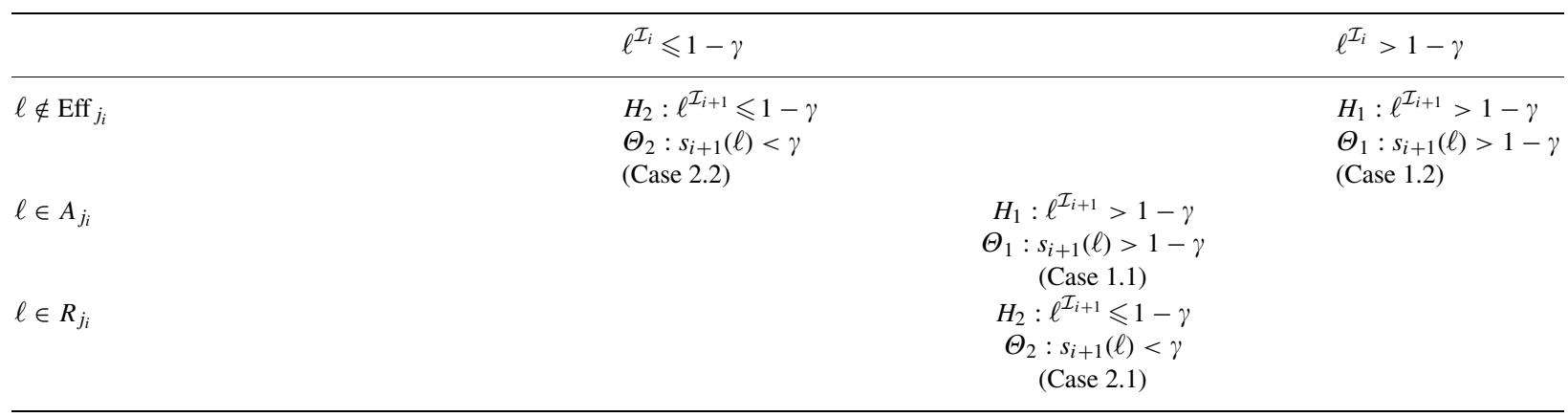

The rows refer to the three possible values of the membership of $\ell$ in the sets $A_{j_{i}}$ and $R_{j_{i}}$; the columns refer to the possible truth values of $\ell$ in world state $\mathcal{I}_{i}$. Each cell contains the hypothesis on the value of $\ell^{\mathcal{I}_{i+1}}$ and the thesis that has to be proven, as well as a reference to the case number in the proof.

To show that such an interpretation $\mathcal{I}_{i+1}$ exists, we construct it: we take the only effect Eff $_{j_{i}}$ of Context $j_{i}$ (i.e., the one such that Context ${\tilde{\mathcal{I}_{i}}}_{j_{i}} \geqslant s_{i}^{\mathcal{I}_{i}}$; ; for all literal $\ell \in \mathcal{L}$,

$$
\ell^{\mathcal{I}_{i+1}}= \begin{cases}\ell^{\mathcal{I}_{i}} & \text { if } \ell \notin \mathrm{Eff}_{j_{i}}, \\ 1 & \text { if } \ell \in A_{j_{i}}, \\ 0 & \text { if } \ell \in R_{j_{i}} .\end{cases}
$$

By Definition 2,

$$
\begin{aligned}
s_{i}^{\mathcal{I}_{i}}>1-\gamma & \Leftrightarrow \min _{\ell} \max \left\{1-s_{i}(\ell), \min \left\{s_{i}(\ell), \ell^{\mathcal{I}_{i}}\right\}\right\}>1-\gamma \\
& \Leftrightarrow \forall \ell\left(1-s_{i}(\ell)>1-\gamma\right) \vee\left(\ell^{\mathcal{I}_{i}}>1-\gamma \wedge s_{i}(\ell)>1-\gamma\right) \\
& \Leftrightarrow \forall \ell\left(s_{i}(\ell)<\gamma\right) \vee\left(\ell^{\mathcal{I}_{i}}>1-\gamma \wedge s_{i}(\ell)>1-\gamma\right) .
\end{aligned}
$$

We recall that, by Definition $8, s_{i+1}$ is constructed such that, for all $\ell \in \mathcal{L}$,

$$
s_{i+1}(\ell)=\max _{j} \min \left\{\max \left\{s_{i}(\ell), A_{j}^{\mathcal{I}_{i}}(\ell)\right\}, 1-R_{j}^{\mathcal{I}_{i}}(\ell)\right\},
$$

where

$$
\begin{aligned}
A_{j}^{\mathcal{I}_{i}}(\ell) & =\min \left\{\text { Context }_{j}^{\mathcal{I}_{i}}, A_{j}(\ell)\right\}, \\
R_{j}^{\mathcal{I}_{i}}(\ell) & =\min \left\{\text { Context }_{j}^{\mathcal{I}_{i}}, R_{j}(\ell)\right\} .
\end{aligned}
$$

We now prove that, for all $\ell \in \mathcal{L}$,

$$
\left(s_{i+1}(\ell)<\gamma\right) \vee\left(\ell^{\mathcal{I}_{i+1}}>1-\gamma \wedge s_{i+1}(\ell)>1-\gamma\right) .
$$

It can be observed that, because of Eq. (41), for all $\ell \in \mathcal{L}$,

$$
\ell^{\mathcal{I}_{i+1}}>1-\gamma \Leftrightarrow\left(\ell \notin \operatorname{Eff}_{j_{i}} \wedge \ell^{\mathcal{I}_{i}}>1-\gamma\right) \vee \ell \in A_{j_{i}} .
$$

To prove Eq. (46), it will suffice to show that

(1) when $\ell^{\mathcal{I}_{i+1}}>1-\gamma$ (let us call this hypothesis $\left.H_{1}\right), s_{i+1}(\ell)>1-\gamma$ (let us call this thesis $\Theta_{1}$ );

(2) when $\ell^{\mathcal{I}_{i+1}} \leqslant 1-\gamma$ (let us call this hypothesis $\left.H_{2}\right), s_{i+1}(\ell)<\gamma$ (let us call this thesis $\Theta_{2}$ ).

To illustrate more clearly the situation, let us consider Table 1 , whose rows refer to the three possible values of the membership of $\ell$ in the sets $A_{j_{i}}$ and $R_{j_{i}}$, and whose columns refer to the possible truth values of $\ell$ in world state $\mathcal{I}_{i}$. Each cell of the table contains the corresponding hypothesis on the value of $\ell^{\mathcal{I}_{i+1}}$ and the thesis that has to be proven. 


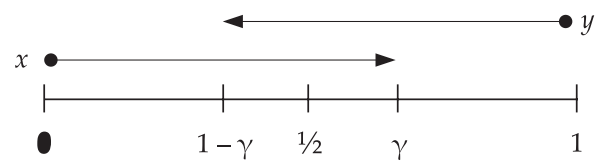

Fig. 2. Diagram showing the values $x<\gamma$ and $y<1-\gamma$ can take up when $\gamma>\frac{1}{2}$.

We examine hereby every case and sub-case, showing that, for each case, the corresponding thesis holds. Case 1.1: If $\ell \in A_{j_{i}}$, we prove $s_{i+1}(\ell)>1-\gamma$. There are two possibilities:

(a) $\ell^{\mathcal{I}_{i}}>1-\gamma$ and thus also, by the induction hypothesis, $s_{i}(\ell)>1-\gamma$.

(b) $\ell^{\mathcal{I}_{i}} \leqslant 1-\gamma$ and thus also, by the induction hypothesis, $s_{i}(\ell)<\gamma$.

Case 1.1(a): In this case, it is sufficient to perform a few calculations:

$$
\begin{aligned}
s_{i+1}(\ell) & \geqslant \min \left\{\max \left\{s_{i}(\ell), A_{j}^{\mathcal{I}_{i}}(\ell)\right\}, 1-R_{j}^{\mathcal{I}_{i}}(\ell)\right\} \\
& >\min \{1-\gamma, 1\}=1-\gamma,
\end{aligned}
$$

which proves $\Theta_{1}$.

Case 1.1(b): In this case, $A_{j_{i}}^{\mathcal{I}_{i}}(\ell)=\min \left\{\right.$ Context $\left._{j_{i}}^{\mathcal{I}_{i}}, 1\right\}=\operatorname{Context}_{j_{i}}^{\mathcal{I}_{i}} \geqslant s_{i}^{\mathcal{I}}>1-\gamma$. It follows that $s_{i+1} \geqslant \min \{\max \{x$, $y\}, 1\}=\max \{x, y\}$, where $x=s_{i}(\ell)<\gamma$ and $y=A_{j_{i}}^{\mathcal{I}_{i}}(\ell)>1-\gamma$. Now, there are three possibilities:

- $\gamma<\frac{1}{2}$ : hence $s_{i+1}(\ell) \geqslant \max \{x, y\}=y>1-\gamma$;

- $\gamma=\frac{1}{2}$ : hence $s_{i+1}(\ell) \geqslant \max \{x, y\}>\frac{1}{2}=\gamma=1-\gamma$;

- $\gamma>\frac{1}{2}$ : hence $s_{i+1}(\ell) \geqslant \max \{x, y\}>1-\gamma$, as it can be concluded from the diagram in Fig. 2, which shows the values $x$ and $y$ can take up with respect to $\gamma$ and $1-\gamma$.

This concludes the proof for Case 1.1.

Case 1.2: In this case, $s_{i+1} \geqslant \min \left\{s_{i}(\ell), 1\right\}=s_{i}(\ell)$, but since, by hypothesis, $\ell^{\mathcal{I}_{i}}>1-\gamma$, it must be also the case that $s_{i}(\ell)>1-\gamma$; therefore, we conclude $s_{i+1}(\ell)>1-\gamma$, and this exhausts the analysis for Case 1 .

Case 2.1: If $\ell \in R_{j_{i}}$, we also know, by hypothesis, that $p e_{j_{i}}=p e_{1}$ is the only effect of action $a, j_{i}=1$, and Context $_{j_{i}}=$ Context $_{1}=\top$ because of Definition 6. As a consequence, Context ${ }_{1}^{\mathcal{I}_{i}}=1, A_{1}^{\mathcal{I}_{i}}(\ell)=0$, and $R_{1}^{\mathcal{I}_{i}}(\ell)=1$. Therefore, we have

$$
\begin{aligned}
s_{i+1}(\ell) & =\min \left\{\max \left\{s_{i}(\ell), A_{1}^{\mathcal{I}_{i}}(\ell)\right\}, 1-R_{1}^{\mathcal{I}_{i}}(\ell)\right\} \\
& =\min \left\{\max \left\{s_{i}(\ell), 0\right\}, 0\right\}=0<\gamma .
\end{aligned}
$$

Case 2.2: In this last case, by hypothesis we know $\ell \notin$ Eff $_{j_{i}}$, thus also $\ell \notin A_{j_{i}}$ and $\ell \notin R_{j_{i}}$. Furthermore, $\ell^{\mathcal{I}_{i}} \leqslant 1-\gamma$ and $\ell^{\mathcal{I}_{i+1}} \leqslant 1-\gamma$. Now, for $\ell^{\mathcal{I}_{i}} \leqslant 1-\gamma$ to hold, it must be $s_{i}(\ell)<\gamma$; in addition, $A_{j_{i}}^{\mathcal{I}_{i}}(\ell)=R_{j_{i}}^{\mathcal{I}_{i}}(\ell)=0$. Therefore, for $j=j_{i}$,

$$
\min \left\{\max \left\{s_{i}(\ell), 0\right), 1-0\right\}=\min \left\{s_{i}(\ell), 1\right\}=s_{i}(\ell)<\gamma .
$$

Now, in order to prove $\Theta_{2}$, it is sufficient to show that for all other $j \neq j_{i}$ as well, we have

$$
\min \left\{\max \left\{s_{i}(\ell), A_{j}^{\mathcal{I}_{i}}(\ell)\right\}, 1-R_{j}^{\mathcal{I}_{i}}(\ell)\right\}<\gamma .
$$

For $j \neq j_{i}$, since $\sum_{j}$ Context $_{j}^{\mathcal{I}_{i}}=1$ and Context $j_{i}>1-\gamma$, it must be Context ${ }_{j}<\gamma$ and, therefore, $A_{j}^{\mathcal{I}_{i}}(\ell)<\gamma$ and $R_{j}^{\mathcal{I}_{i}}(\ell)<\gamma$. As a consequence, $\max \left\{s_{i}(\ell), A_{j}^{\mathcal{I}_{i}}(\ell)\right\}<\gamma$, because both its arguments are $<\gamma$, whence $\min \left\{\max \left\{s_{i}(\ell)\right.\right.$, $\left.\left.A_{j}^{\mathcal{I}_{i}}(\ell)\right\}, 1-R_{j}^{\mathcal{I}_{i}}(\ell)\right\}<\gamma$. This proves $\Theta_{2}$, completes the proof of Case 2.2, as well as of the induction step.

To sum up, we have proved that if there exists an interpretation $\mathcal{I}_{0}$ such that $s_{0}^{\mathcal{I}_{0}}>1-\gamma$, one can construct a sequence of world states $\left\{\mathcal{I}_{i}\right\}_{i=0, \ldots, N}$, and a trajectory $\left\langle s_{0}, \ldots, s_{N}\right\rangle$ such that, for all $i, s_{i}^{\mathcal{I}_{i}}>1-\gamma$. Therefore, for all $i, \pi\left(s_{i+1} \mid s_{i}, a_{i}\right)>1-\gamma$, which, by Proposition 8, implies $G\left(s_{N}\right) \geqslant \gamma$, which is the thesis. 


\subsection{Equivalence}

In this section we will show that solving a fuzzy possibilistic planning is equivalent, under certain conditions, to solving a possibilistic planning problem.

Definition 22 (Complete state description). A complete state description is a state description $s$ such that, for all atom $p \in \mathcal{A}$, either $s(p)=1$ or $s(\neg p)=1$ (but not both).

In our formalism, it is easy to verify that, given a complete state description, there exists exactly one crisp interpretation that satisfies it, and vice versa.

Definition 23. A complete state description corresponding to a state description $s$ is a state description $s^{\prime}$ such that for all $p$ :

- $s^{\prime}(p)=1$ if $s(p)>s(\neg p)$;

- $s^{\prime}(p)=0$ if $s(p) \leqslant s(\neg p)$.

In [7], a (crisp) possibilistic planning problem $\Delta^{\prime}$ has been defined as a triple $\left\langle s_{0}^{\prime}, \varepsilon_{G}, A\right\rangle$; where $s_{0}^{\prime}$ is a complete description of the initial state, $\varepsilon_{G}$ is a conjunction of literals, and $A$ is a set of available possibilistic actions. Therefore, a state $s$ may or may not belong to the set of states satisfying the expression $\varepsilon_{G}$. If $G$ is the set of states satisfying the goal, a plan $P$ is said to be $\gamma$-acceptable for $\Delta^{\prime}$ if and only if the necessity of its reaching the goal is greater than $\gamma$, i.e., if a final state $s_{N}$ is such that $\forall\left\langle s_{1} \ldots s_{N}\right\rangle, \forall i \pi\left[s_{i+1} \mid s_{i}, a_{i}\right]>1-\gamma, s_{N} \in G$. One of the most important results of that work states that the search for a $\gamma$-acceptable plan amounts to solving a planning problem straightforwardly derived from the original possibilistic one and consisting only of pure (nongraded) nondeterministic actions.

Definition 24. A possibilistic planning problem $\Delta^{\prime}=\left\langle s_{0}^{\prime}, \varepsilon_{G}, A\right\rangle$ is the crisp counterpart of a fuzzy possibilistic planning problem $\Delta=\left\langle s_{0}, \varepsilon_{G}, A\right\rangle$ if and only if $s_{0}^{\prime}$ is a complete description corresponding to $s_{0}$.

Proposition 10. Let $\Delta^{\prime}=\left\langle s_{0}^{\prime}, \varepsilon_{G}, A\right\rangle$ be a crisp counterpart of the fuzzy possibilistic planning problem $\Delta=\left\langle s_{0}, \varepsilon_{G}, A\right\rangle$ such that $\varepsilon_{G}$ is a conjunction of literals. If a sequential plan $P$ is $\gamma$-acceptable for $\Delta^{\prime}, P$ is also $\gamma$-acceptable for its fuzzy counterpart.

Proof. By definition, $\mathrm{P} \gamma$-acceptable for a (crisp) counterpart $\Delta^{\prime}$ of the fuzzy possibilistic planning problem $\Delta$ is equivalent to

$$
\forall\left\langle s_{0}^{\prime} \ldots s_{N}^{\prime}\right\rangle, \forall i \pi\left[s_{i+1}^{\prime} \mid s_{i}^{\prime}, a_{i}\right]>1-\gamma \Rightarrow s_{N}^{\prime} \in G .
$$

Each $s_{i}^{\prime}$ is a complete world state corresponding to an incomplete state description $s_{i}$. Let $\mathcal{I}_{i}$ be the crisp interpretation that satisfies $s_{i}^{\prime}$. Then,

$$
\begin{aligned}
\mathrm{P} \gamma \text {-acceptable for } \Delta^{\prime} & \Leftrightarrow \forall\left\langle\mathcal{I}_{0} \ldots \mathcal{I}_{N}\right\rangle, \forall i \pi\left[\mathcal{I}_{i+1} \mid \mathcal{I}_{i}, a_{i}\right]>1-\gamma \Rightarrow \mathcal{I}_{N} \in G \\
& \Leftrightarrow \forall\left\langle\mathcal{I}_{0} \ldots \mathcal{I}_{N}\right\rangle, \forall i \pi\left[\mathcal{I}_{i+1} \mid \mathcal{I}_{i}, a_{i}\right]>1-\gamma \Rightarrow G\left(\mathcal{I}_{N}\right) \geqslant \gamma \in(0,1] .
\end{aligned}
$$

Each $\mathcal{I}_{i}$ satisfies a possible complete representation of its counterpart in $\Delta$. Therefore, if we note $\left\langle s_{0} \ldots s_{N}\right\rangle$ a trajectory of incomplete state descriptions such that each $s_{i}$ has a corresponding complete state description satisfied by crisp interpretation $\mathcal{I}_{i}$, the above equivalence may be written as

$$
\begin{aligned}
& \mathrm{P} \gamma \text {-acceptable for } \Delta^{\prime} \\
& \quad \Leftrightarrow \forall\left\langle s_{0} \ldots s_{N}\right\rangle, \exists\left\langle\mathcal{I}_{0} \ldots \mathcal{I}_{N}\right\rangle \text { such that } \forall i \pi\left[\mathcal{I}_{i+1} \mid \mathcal{I}_{i}, a_{i}\right]>1-\gamma \Rightarrow G\left(s_{N}\right) \geqslant \gamma \in(0,1] \\
& \quad \Leftrightarrow \forall\left\langle s_{0} \ldots s_{N}\right\rangle, \forall i \pi\left[s_{i+1} \mid s_{i}, a_{i}\right]>1-\gamma \Rightarrow G\left(s_{N}\right) \geqslant \gamma \in(0,1] \\
& \quad \Leftrightarrow \mathrm{P} \gamma \text {-acceptable for } \Delta . \quad \square
\end{aligned}
$$

This result allows us to write the fuzzy posplan algorithm FPOSPLAN. 


\author{
Algorithm $\operatorname{FPOSPLAN}(\Delta, \gamma)$ \\ (1) transform $\Delta$ into a (crisp) possibilistic planning problem $\Delta^{\prime}$; \\ (2) return $\mathcal{P} \leftarrow P O S P L A N\left(\Delta^{\prime}, \gamma\right)$
}

Fig. 3. FPOSPLAN: general algorithm.

\title{
6.4. A fuzzy planning algorithm for $\gamma$-acceptability
}

Given a fuzzy possibilistic planning problem $\Delta$, we proposed above a way to transform it into a (crisp) possibilistic planning problem $\Delta^{\prime}$. This transformation is the core of our fuzzy possibilistic planning algorithm called FPOSPLAN (Fig. 3). The possibilistic planning algorithm POSPLAN, described in [7], takes a possibilistic planning problem $\Delta^{\prime}=\left\langle s_{0}^{\prime}, \varepsilon_{G}, A\right\rangle$ as input, and generates a plan $P$, which is $\gamma$-acceptable for $\Delta$.

This result of equivalence between (crisp) possibilistic planning $\Delta^{\prime}$ and fuzzy possibilistic planning $\Delta$ establishes that, under certain conditions, the complexities of these two problems are equivalent and, as a consequence, lower than the complexity of probabilistic planning problems, as shown in [8].

\section{Conclusions}

The fundamental definitions for approaching possibilistic planning when knowledge about the world and actions is represented by means of fuzzy state descriptions have been provided, and the possibilistic planning problem has been recast in this setting.

We have chosen to represent the planning agent's incomplete and imprecise knowledge of the world by means of neutrosophic fuzzy sets. We have accordingly defined a model-theoretic semantics for such fuzzy state descriptions and proved some useful properties.

Then we have defined fuzzy possibilistic actions and their semantics, and we have examined basic reasoning tasks regarding actions and their effects. Next action plans have been considered and characterized.

The notion of a fuzzy possibilistic planning problem, i.e., a possibilistic planning problem with imprecise and incomplete knowledge, has been introduced and characterized. The notion of $\gamma$-acceptability of a solution plan, previously defined for crisp possibilistic planning has been extended to this new framework of fuzzy possibilistic planning, and sufficient conditions for the existence of solutions for fuzzy possibilistic planning problems have been derived and proved.

Finally, we have shown that, under certain conditions, solving a fuzzy possibilistic planning problem can be reduced to solving a crisp possibilistic planning problem.

\section{References}

[1] K. Atanassov, Intuitionistic fuzzy sets, Fuzzy Sets and Systems 20 (1986) 87-96.

[2] F. Bacchus, R. Petrick, Modeling an agent's incomplete knowledge during planning and execution, in: A.G. Cohn, L. Schubert, S.C. Shapiro (Eds.), KR'98: Principles of Knowledge Representation and Reasoning, Morgan Kaufmann, San Francisco, CA, 1998, pp. $432-443$.

[3] S. Benferhat, D. Dubois, S. Kaci, H. Prade, Modeling positive and negative information in possibility theory, International Journal of Intelligent Systems 23 (10) (2008) 1094-1118.

[4] A.L. Blum, M.L. Furst, Fast planning through planning graph analysis, Artificial Intelligence 90 (1997) 281-300.

[5] D. Chapman, Planning for conjunctive goals, Artificial Intelligence 32 (3) (1987) 333-377.

[6] C. Cornelis, C. van der Donck, E. Kerre, Sinha-Dougherty approach to the fuzzification of set inclusion revisited, Fuzzy Sets and Systems 134 (2003) 283-295.

[7] C. da Costa Pereira, F. Garcia, J. Lang, R. Martin-Clouaire, Planning with graded nondeterministic actions: a possibilistic approach, International Journal of Intelligent Systems 12 (1997) 935-962.

[8] C. da Costa Pereira, F. Garcia, J. Lang, R. Martin-Clouaire, Possibilistic planning: representation and complexity, in: ECP, 1997 , pp. $143-155$.

[9] D. Dubois, H. Prade, Possibility Theory-An approach to Computerized Processing of Uncertainty, Plenum Press, New York, 1988.

[10] D. Dubois, H. Prade, An introduction to bipolar representations of information and preference, International Journal of Intelligent Systems 23 (8) (2008) 866-877.

[11] T. Eiter,W. Faber, N. Leone, G. Pfeifer, A. Polleres, Planning under Incomplete Knowledge, in: Lecture Notes in Computer Science, Vol. 1861, Springer, Berlin, 2000, pp. 807-821.

[12] R. Fikes, N. Nilsson, Strips: a new approach to the application of theorem proving to problem solving, Artificial Intelligence 2 (1971) 189-208.

[13] A. Finzi, F. Pirri, R. Reiter, Open world planning in the situation calculus, in: AAAI/IAAI, 2000, pp. 754-760. 
[14] F. Giunchiglia, Planning as satisfiability with expressive action languages: concurrency, constraints and nondeterminism, in: Proc. Seventh Internat. Conf. on Principles of Knowledge Representation and Reasoning (KR'00), 2000.

[15] L. Iocchi, D. Nardi, R. Rosati, Strong cyclic planning with incomplete information and sensing, in: Proc. 4th Internat. Workshop on Planning and Scheduling for Space, Darmstadt, Germany, 2004.

[16] N. Kushmerick, S. Hanks, D.S. Weld, An algorithm for probabilistic planning, Artificial Intelligence 76 (1-2) (1995) $239-286$.

[17] N. McCain, H. Turner, Satisfiability planning with causal theories, in: A.G. Cohn, L. Schubert, S.C. Shapiro (Eds.), KR'98: Principles of Knowledge Representation and Reasoning, Morgan Kaufmann, San Francisco, CA, 1998, pp. 212-223.

[18] J.S. Penberthy, D.S. Weld, UCPOP: a sound, complete, partial order planner for ADL, in: B. Nebel, C. Rich, W. Swartout (Eds.), Principles of Knowledge Representation and Reasoning: Proc. Third Internat. Conf. (KR'92), Kaufmann, San Mateo, CA, 1992, pp. $103-114$.

[19] M. Peot, D. Smith, Conditional nonlinear planning, in: J. Hendler (Ed.), Proc. First Internat. Conf. on AI Planning Systems, Morgan Kaufmann, College Park, MD, 1992, pp. 189-197.

[20] R. P. A. Petrick, F. Bacchus, A knowledge-based approach to planning with incomplete information and sensing, in: AI Planning and Scheduling, 2002, pp. 212-222.

[21] L. Pryor, G. Collins, Planning for contingencies: a decision-based approach, Journal of Artificial Intelligence Research 4 (1996) $287-339$.

[22] J. Rintanen, Constructing conditional plans by a theorem-prover, Journal of Artificial Intelligence Research 10 (1999) 323-352.

[23] U. Rivieccio, Neutrosophic logics: prospects and problems, Fuzzy Sets and Systems 159 (14) (2008) 1860-1868.

[24] A. Saffiotti, Handling uncertainty in control of autonomous robots, in: Applications of Uncertainty Formalisms, Springer, London, UK, 1998, pp. 198-224.

[25] F. Smarandache, A unifying field in logics: neutrosophic logic, Multiple-Valued Logic 8 (3) (2002) 385-438.

[26] A. Tate, Generating project networks, in: Proc. Fifth IJCAI, 1977, pp. 888-893.

[27] H. Wang, R. Sunderraman, A data model based on paraconsistent intuitionistic fuzzy relations, in: M.-S. Hacid, N.V. Murray, Z.W. Ras, S. Tsumoto (Eds.), Foundations of Intelligent Systems, Proc. 15th Internat. Symp., ISMIS 2005, Saratoga Springs, NY, USA, Lecture Notes in Computer Science, Vol. 3488, Springer, Berlin, May 25-28, 2005, pp. 669-677.

[28] D.S. Weld, C.R. Anderson, D.E. Smith, Extending graphplan to handle uncertainty and sensing actions, in: AAAI/IAAI, 1998, pp. 897-904.

[29] L.A. Zadeh, The calculus of fuzzy if-then rules, AI Expert 7 (3) (1992) 22-27. 